\title{
Which Infectious Blood Donors Could Be Identified by the Donor History Questionnaire? - Comparison of Blood Donors Infected with HIV or HCV with Notified Cases from General Population in Germany
}

\author{
Karina Preußel Ruth Offergeld \\ Department for Infectious Disease Epidemiology, Robert Koch Institute, Berlin, Germany
}

\section{Keywords}

Transfusion-transmissible infections - Donor selection . Risk factors

\section{Summary}

Background: Potential risks for transfusion-transmissible infections are identified by donor history questionnaires (DHOs), and donors with higher risks are deferred from donation. We assessed to which extent the currently used DHQs support the identification of infections among blood donors. Methods: Between 2006 and 2013, we analyzed data from notified HIV and HCV cases in the general population and positive blood donors in Germany. Logistic regressions were used to identify relevant infection risks. We estimated the possible effect of improved capture of risk factors for donor selection by calculation of population attributable fractions (PAF). Results: Risky sexual contacts - MSM as well as heterosexual contacts were the most prominent infection risks among HIV-infected donors. Whereas MSM contacts were significantly less reported by donors than by cases from the general population, $58 \%$ of donors disclosed heterosexual risks compared to $26 \%$ of notified cases. The complete identification of heterosexual risk contacts might prevent acceptance of $53 \%$ of HIV-infected donors. HCV-infected donors were more likely to report heterosexual exposure, imprisonment, and piercing/tattoo than notified HCV cases. Improved recording of piercing/tattoo could prevent acceptance of $16 \%$ of HCV-infected donors. Conclusion: Donor selection should be improved with special attention to the identification of (hetero)sexual risk factors, invasive procedures (piercing/tattoo and imprisonment) applying welldesigned DHQs, effective donor education, and confidential environment in all steps of the selection process.

(C) 2018 S. Karger GmbH, Freiburg

\section{Introduction}

In Germany, on average close to 7 million whole blood, plasma, and cytapheresis donations are collected each year. To ensure their safety a careful selection of blood donors is needed. Potential risks for transfusion-transmissible infectious diseases (TTI) are usually identified by donor history questionnaires (DHQ) and donors who pose higher risks are deferred from donation. Successful rejection of infected applicant donors requires identification of possible risk factors using plain and comprehensive questions. Moreover, disclosure of risk behavior is substantially needed. An appropriate pre-donation education for donors should improve their awareness and may support the acceptance of putatively embarrassing questions about risk behavior.

Effectiveness of those measures was previously assessed by comparing prevalence of infections in (first-time) donors with prevalence in the general population. In the Netherlands, e.g., an approximately 20 times lower HCV prevalence and a 60 times lower HIV prevalence was observed in first-time donors compared to the general population. This was related to an effective selection process and responsible self-deferral [1]. In Australia, the HCV prevalence in first-time donors was at least 12 times lower compared to the general population and the HIV prevalence 140 times lower - probably due to a combination of donor education and donor selection [2]. However, these mechanisms seem to be less effective in Germany - HCV prevalence in first-time donors was only reduced by a factor of 5 (61/100,000 first-time donors vs. $300 / 100,000$ general population (18-79 years) [3]), and HIV prevalence was reduced by a factor of 20 (5.8/100,000 vs. 136/100,000 (20-69 years) [4]). Moreover, in 2013 HCV infections were more frequently detected in blood donors than in the general population, aged $20-69$ years $(11.7 / 100,000$ vs. $8.5 / 100,000)$. Insufficient recording of risk factors as well as donors' non-compliance with selection criteria may contribute to admission of infected persons to blood donation.

\section{KARGER}

(c) 2018 S. Karger GmbH, Freiburg

Fax +497614520714 
Investigations of non-compliance with selection criteria usually focused on MSM $[5,6]$, as sexual contacts between men are still the most important risk factor for HIV infections in non-endemic countries [7-9]. However, the extent of non-disclosure or unawareness of other risk factors is so far unknown in Germany.

Risk factors for transfusion-relevant infections were previously identified in the post-donation interview [1] or by comparing noninfected with infected donors $[7,10]$. In contrast, we aimed to assess to what degree the currently used pre-donation DHQs support the deferral of infected individuals by comparing transmission risks of donors with those of infected persons from the general population. Risk factors that need improvement in their recording were identified, and the impact of their improved recording was estimated.

\section{Material and Methods}

\section{Study Design}

We performed a retrospective analysis, which included all notified HIV and HCV cases aged 18-68 years and all HIV-or HCV-infected blood donors in Germany with recorded transmission risks between 2006 and 2013.

Notified HIV or HCV cases from the general population were included in the study if they fulfilled the case definitions. Blood donors were eligible for the study if they were found to be positive for HIV or HCV in the context of a blood donation. Cases and blood donors were excluded if probable risk factors were not recorded.

Pre-donation interviews and examinations for selection of donors are equally performed for both first-time and repeat donors in Germany (no abbreviated DHQs in use). Therefore, we did not stratify our analysis for these subpopulations.

\section{Data Source}

HIV and HCV infections are notifiable in Germany according to the Infections Protection Act (general population) and the Transfusion Act (blood donors) and were anonymously reported to the national surveillance databases, which are hosted by the Robert Koch Institute (RKI), Germany's federal public health institute.

\section{Case Definition}

General population: HIV infections were notifiable if the infection was diagnosed by detection of HIV antibodies in serum or plasma and confirmation with western blotting. HCV infections were notifiable if the infection was diagnosed by detection of HCV antibodies in serum or plasma and confirmation with immunoblot or if a nucleic acid test (NAT) was positive.

Blood donors: HIV infections were notifiable if the infection was diagnosed by detection of HIV antibodies in serum or plasma and confirmed with western blotting or detection of viral nucleic acid. HCV infections were notifiable if the infection was diagnosed by detection of HCV antibodies in serum or plasma and confirmed with immunoblot or by positive NAT .

\section{Data Analysis}

Age groups (18-24 years; $25-34$ years; $35-44$ years; $45-54$ years; $55+$ years) were created for reports from the general population in order to resemble the age classes used for blood donor surveillance reports in Germany. Residence was classified as metropolis if the blood establishment reported residence of an infected blood donor in a city $>100,000$ inhabitants or if an infection of an individual in the general population was reported by local health department of a metropolis (>100,000 inhabitants).

Infected blood donors and infected persons from the general population were described with respect to demographic characteristics. Statistical signifi- cances of demographic and risk-specific differences between blood donors and the general population were checked by means of $\chi^{2}$ test. Age differences were checked using the Wilcoxon rank-sum test.

Recording of HIV transmission risks differed between blood donors and the general population. The categories MSM contact, heterosexual risk contact, intravenous drug use and occupational exposure are used for both types of notification (blood donors and the general population). For blood donors, additional exposure categories were available like piercing/tattoo, sex work, household exposure, and imprisonment. The category 'intravenous drug use' also included nasal drug use. These were not recorded separately for HIV-infected subjects in the general population and therefore, summed up in the category 'others'. Multiple answers were allowed.

Weighted proportions of possible transmission risks were given considering multiple (competing) risk reports. Infected persons who reported $n$ possible transmission risks contributed with $1 / n$ to the total proportion of a particular risk factor.

Univariable analysis was performed for all transmission risks. We estimated univariable odds ratios (OR) and 95\% confidence intervals (95\% CI) using logistic regression with adjustment for the probable confounding effects of age group, sex, and type of residence.

Multivariable logistic regression modeling was used to identify transmission risks which describe infected blood donors compared to the general infected population. We employed a forward stepwise selection strategy with the forcedin confounding variables and transmission risks as candidate variables that had a p-value $<0.05$ in univariable analysis.

Population-attributable fractions (PAFs) are usually estimated based on multivariable models to quantify the proportion of (ill) cases that can be attributed to one or more specified risk factors. They describe the reduction in the population affected that would occur if particular risk factors are completely absent. In the context of our study, PAFs were used to estimate the possible effect of improved capture of infection risks on the admission of infected donors. They represent the (theoretical) reduction of infections if the responsible risk factor was identified completely by DHQs. PAFs for transmission risks in the final multivariable model were calculated with $95 \%$ CI based on the method described by Greenland and Drescher [11] and Newsom [12].

We performed a sensitivity analysis and used a multiple imputation method to assess the possible impact of missing transmission risk data to our analysis. Based on statistical associations between demographic data and several transmission risks, (plausible) values were imputed for each transmission risk. The sensitivity analysis was performed with a data set including all infected blood donors and infected persons from the general population which were excluded from the study due to non-recording of infection risks.

All statistical analyses were performed using STATA/SE 14.0 (StataCorp LP, College Station, TX, USA).

\section{Results}

\section{HIV}

Between 2006 and 2013 22,044 new HIV diagnoses in the general population (HIV+ GP) and 789 HIV infections among blood donors (HIV+ BD) were reported to the national databases. Information about transmission risks were available for 309 blood donors (39\%) and 17,366 persons in the general population $(79 \%$, $\mathrm{p}<0.001)$.

$\mathrm{HIV}+\mathrm{BD}$ with reported transmission risks differed from those without reported risks: HIV+ BD that were included in the analysis were significantly younger (median age 28 years vs. 33 years, $\mathrm{p}<0.001)$ and lived more frequently in metropolis $(60 \%$ vs. $51 \%$, $p=0.013)$ than excluded HIV+ BD. Enrolled HIV+ GP were also significantly younger (median age 36 years vs. 38 years; $\mathrm{p}<0.001$ ), 
Table 1. Characteristics of individuals (blood donors and general population) with HIV infections and reported transmission risks, 2006-2013

\begin{tabular}{|c|c|c|c|c|c|c|c|}
\hline & \multicolumn{2}{|c|}{$\begin{array}{l}\text { Blood donors } \\
(\mathrm{n}=309)\end{array}$} & \multicolumn{2}{|c|}{$\begin{array}{l}\text { General population } \\
(\mathrm{n}=17,366)\end{array}$} & \multirow[t]{2}{*}{$\mathrm{p}$} & \multirow[t]{2}{*}{$\mathrm{aOR}^{*}$} & \multirow[t]{2}{*}{$95 \% \mathrm{CI}$} \\
\hline & $\mathrm{n}$ & $\%$ & $\mathrm{n}$ & $\%$ & & & \\
\hline Age, years & & & & & $<0.001$ & & \\
\hline $18-24$ & 105 & 34.0 & 2,031 & 11.7 & & & \\
\hline $25-34$ & 119 & 38.5 & 5,834 & 33.6 & & & \\
\hline $35-44$ & 47 & 15.2 & 5,556 & 32.0 & & & \\
\hline $45-54$ & 26 & 8.4 & 2,915 & 16.8 & & & \\
\hline $55+$ & 12 & 3.9 & 1,030 & 5.9 & & & \\
\hline Male sex & 266 & 86.1 & $15,190^{1}$ & 87.7 & 0.38 & & \\
\hline Metropolis & $183^{2}$ & 60.0 & 12,057 & 69.4 & $<0.001$ & & \\
\hline MSM & 133 & 43.0 & 12,418 & 71.5 & $<0.001$ & 0.2 & $0.1-0.2$ \\
\hline Heterosexual risk contact & 178 & 57.6 & 4,534 & 26.1 & $<0.001$ & 6.6 & $5.1-8.5$ \\
\hline Intravenous drug use & 8 & 2.6 & 900 & 5.2 & 0.04 & 0.5 & $0.2-0.9$ \\
\hline Occupational exposure & 19 & 6.2 & 5 & $<0.1$ & $<0.001$ & 196.7 & $69.7-555.7$ \\
\hline Other & 71 & 23.0 & 5 & $<0.1$ & $<0.001$ & 904.0 & $357.5-2,285.9$ \\
\hline
\end{tabular}

Fig. 1. Probable mode of transmission of HIV infections reported by infected blood donors (black) and infected persons from the general population (grey), 2006-2013. Solid - proportion of unique risk reports; striped - proportion of reports depending on number of multiple answers.

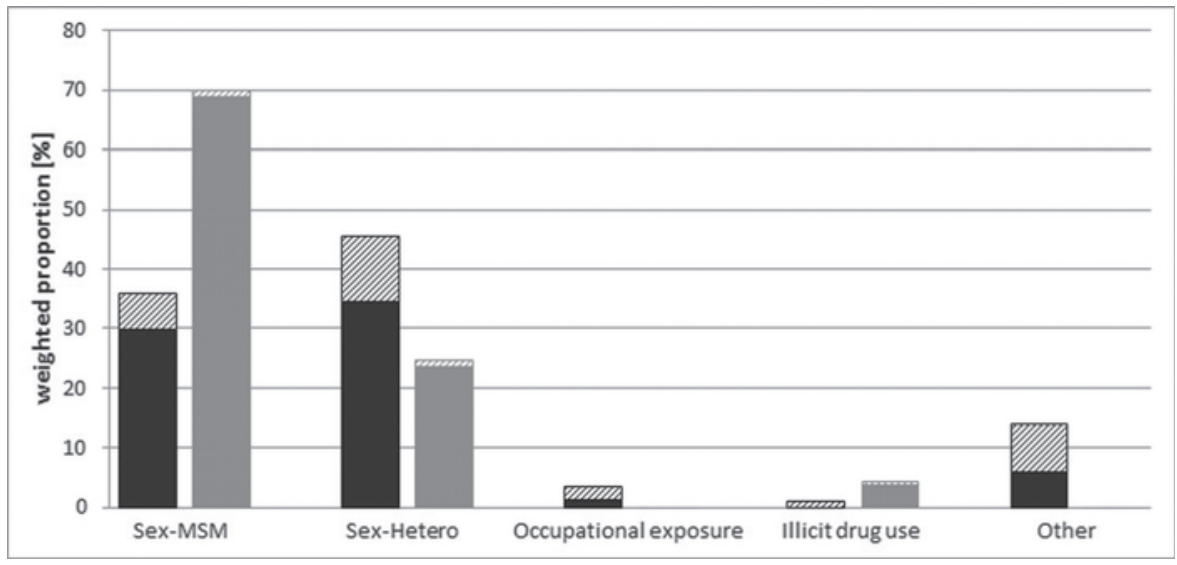

lived in a metropolis more frequently ( $69 \%$ vs. $59 \%, \mathrm{p}<0.001)$, and were more often male ( $88 \%$ vs. $69 \%, \mathrm{p}<0.001)$ compared to excluded HIV+ GP.

$\mathrm{HIV}+\mathrm{BD}$ and $\mathrm{HIV}+\mathrm{GP}$ with reported transmission risks were similar with respect to sex but were significantly younger (median age 28 years vs. 36 years; $p<0.001$ ) and lived in a metropolis less frequently than HIV+ GP. Demographic characteristics of both populations are listed in table 1.

Sexual contacts were the most prominent reported risk factors among HIV+ GP as well as HIV+ BD - 285 HIV+ BD (92\%) and $16,675 \mathrm{HIV}+\mathrm{GP}(96 \%)$ reported either MSM or heterosexual risk contacts, or both. When more than one risk was reported, weighted proportions for sexual risks were $82 \%$ for $\mathrm{HIV}+\mathrm{BD}$ and $95 \%$ for $\mathrm{HIV}+\mathrm{GP}$. HIV+ BD had a significantly higher proportion of heterosexual risk contacts compared to HIV+ GP, whereas HIV+ GP reported MSM significantly more often than HIV+ BD (fig. 1, table 1). Furthermore, occupational exposure and 'other' transmission risks were reported significantly more often by HIV+ BD than by HIV+ GP. In contrast, drug use was significantly more frequent among HIV+ GP. Multiple risks were recorded 10 times more frequently in HIV+ BD than in HIV+ GP (29\% vs. $3 \%$, p < 0.001$)$.

All recorded risk factors were significantly associated (negatively or positively) with the status 'blood donor' in univariable analysis adjusted for the confounding effects of age, sex, and residence (table 1).

The final multivariable model included MSM, heterosexual risk contact, occupational exposure, and others as transmission risks with significant positive association to blood donors. (table 2). The PAFs for the single transmission risks range from 6\% (occupational exposure) to $54 \%$ (heterosexual risk contacts).

The sensitivity analysis confirmed the results of the complete case analysis: heterosexual risk contacts, occupational exposure, and 'other' transmission risks were found to be significantly associated with the status blood donor with an OR $>1(\mathrm{p}<0.001)$ in the univariable analysis of transmission risks and could be identified as risk factors for underestimation in blood donors by multivariable analysis as well. Estimated PAFs were comparable (heterosexual risk contacts $48 \%$, occupational exposure $8 \%$, 'other' transmission risks $25 \%$ ). 
Table 2. Multivariable model for risk factors describing HIV-infected blood donors

\begin{tabular}{lrcccc}
\hline & OR & $95 \%$ CI & p & PAF, \% & $95 \%$ CI, \% \\
\hline Age, years* & & & & & \\
$18-24$ & 7.4 & $3.7-15.0$ & $<0.001$ & & \\
$25-34$ & 2.7 & $1.3-5.4$ & 0.01 & & \\
$35-44$ & 1.2 & $0.6-2.6$ & 0.57 & & \\
$45-54$ & 1.1 & $0.5-2.4$ & 0.85 & & \\
$\quad 55+$ & ref & & & & \\
Sex female & 0.3 & $0.2-0.4$ & $<0.001$ & & \\
Metropolis* & 0.9 & $0.7-1.2$ & 0.42 & & \\
MSM & 1.9 & $1.2-3.0$ & 0.01 & 19.9 & $8.7-29.7$ \\
Heterosexual risk contact & 11.3 & $7.2-17.9$ & $<0.001$ & 53.2 & $50.8-55.5$ \\
Occupational exposure & 345.7 & $103.7-1,152.0$ & $<0.001$ & 6.2 & $6.2-6.2$ \\
Other & 1681.6 & $599.7-4,715.8$ & $<0.001$ & 22.9 & $22.9-22.9$ \\
\hline *Adjustment variable. & & & & & \\
\hline
\end{tabular}

Table 3. Characteristics of individuals (blood donors and general population) with HCV infections and reported transmission risks, 2006-2013

\section{$\mathrm{HCV}$}

Between 2006 and 2013, data on transmission risks were available for 674 out of 3,469 (19\%) notified HCV cases among blood donors (HCV+ BD) and for 22,707 out of 41,237 (55\%) notified $\mathrm{HCV}$ cases in the general population $(\mathrm{HCV}+\mathrm{GP} ; \mathrm{p}<0.001)$. $\mathrm{HCV}+\mathrm{BD}$ and $\mathrm{HCV}+\mathrm{GP}$ with reported transmission risks differed from those without reported risks: $\mathrm{HCV}+\mathrm{BD}$ with reported risks were significantly younger (median age 33 years vs. 37 years, $\mathrm{p}<0.001)$ and lived in a metropolis more frequently than excluded HCV + BD (57\% vs. $41 \%, \mathrm{p}<0.001)$ Enrolled HCV+ GP were also significantly younger (median age 38 years vs. 42 years, $\mathrm{p}<0.001$ ) but lived less frequently in a metropolis ( $39 \%$ vs. $41 \%, \mathrm{p}<0.001)$.

$\mathrm{HCV}+\mathrm{BD}$ and $\mathrm{HCV}+\mathrm{GP}$ with reported transmission risks were similar with respect to sex distribution (table 3) but HCV+
BD were significantly younger (median age 33 years vs. 38 years; $\mathrm{p}<0.001$ ) and lived in a metropolis more frequently.

Whereas drug use was the most prominent risk factor among $\mathrm{HCV}+\mathrm{GP}$ (weighted proportion 41\%), nosocomial exposure represented the largest proportion in HCV+ BD (33\%, fig. 2). Transmission risks that were significantly more often reported by $\mathrm{HCV}+$ $\mathrm{BD}$ were: heterosexual risk contacts, imprisonment ( $>3$ days), piercing/tattoo, occupational exposure, and nosocomial exposure. In contrast, drug use was significantly less frequently reported by $\mathrm{HCV}+\mathrm{BD}$. No differences were found for reporting of MSM contacts and household exposure $(\mathrm{HCV}+$ person in the same household).

Competing transmission risks were reported more often by HCV+ BD (39\% vs. $34 \%, \mathrm{p}=0.01)$. 
Fig. 2. Probable mode of transmission of $\mathrm{HCV}$ infections reported by infected blood donors (black) and infected persons from the general population (grey), 2006-2013. Solid - proportion of unique risk reports; striped - proportion of reports depending on number of multiple answers.

Table 4. Multivariable model for risk factors describing $\mathrm{HCV}$-infected blood donors

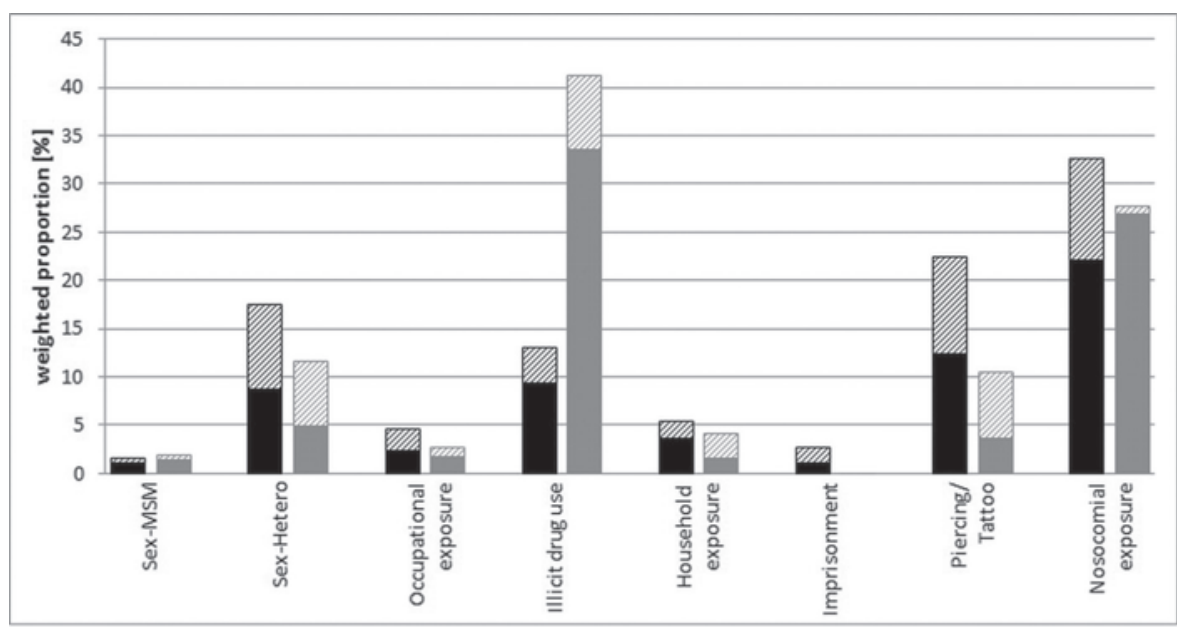

\begin{tabular}{|c|c|c|c|c|c|}
\hline & OR & $95 \% \mathrm{CI}$ & $\mathrm{p}$ & PAF, $\%$ & $95 \% \mathrm{CI}, \%$ \\
\hline \multicolumn{6}{|l|}{ Age, years* } \\
\hline $18-24$ & 14.8 & $9.7-22.7$ & $<0.001$ & & \\
\hline $25-34$ & 5.6 & $3.7-8.5$ & $<0.001$ & & \\
\hline $35-44$ & 3.1 & $2.0-4.8$ & $<0.001$ & & \\
\hline $45-54$ & 3.5 & $2.3-5.3$ & $<0.001$ & & \\
\hline $55+$ & ref & & & & \\
\hline Sex ${ }^{*}$ female & 0.8 & $0.7-1.0$ & 0.02 & & \\
\hline Metropolis* & 2.2 & $1.9-2.6$ & $<0.001$ & & \\
\hline Heterosexual risk contact & 1.3 & $1.1-1.5$ & 0.01 & 6.4 & $2.0-10.7$ \\
\hline Intravenous drug use & 0.1 & $0.1-0.1$ & $<0.001$ & & \\
\hline Imprisonment & 57.5 & $31.8-104.0$ & $<0.001$ & 5.4 & $5.4-5.4$ \\
\hline Piercing/tattoo & 1.7 & $1.5-2.1$ & $<0.001$ & 15.9 & $12.1-19.5$ \\
\hline
\end{tabular}

The univariable analysis of risk factors showed significant associations between the status 'blood donor' and the risk factors heterosexual risk contacts, imprisonment ( $>3$ days), piercing/tattoo, occupational exposure, nosocomial exposure, and drug use (table 3).

The factors imprisonment ( $>3$ days), piercing/tattoo, heterosexual risk contact, and drug use remained significantly associated with $\mathrm{BD}$ in the final multivariable model that additionally included the significant interaction between sex and age group. PAFs of positively associated risk factors were $5.4 \%$ for imprisonment, $6.6 \%$ for heterosexual risk factors, and $16.6 \%$ for piercing/tattoo (table 4 ).

The sensitivity analysis confirmed positive association (OR $>1$; $\mathrm{p}<0.01$ ) of the transmission risks imprisonment, piercing/tattoo, and occupational exposure with blood donors in the univariable analysis.

\section{Discussion}

Donor selection and identification of risk factors for TTI is an important step in maintaining the high level of blood safety. Still, a varying percentage of donors are identified with HIV and HCV in- fections with deferrable risks that were not detected or reported in the donor selection process $[1,5,13,14]$. Comparison of risk factors among infected blood donors and infected individuals from the general population is only rarely used to assess the effectiveness of blood donor selection procedures. Risk factors for TTI and efficiency of donor selection are usually determined within the population of accepted donors [7, 15, 16]. However, we decided to use general population as an assessment reference for donor selection, since infected applicant donors are part of the general population but not part of the accepted donor population. By comparing risk factors for HIV and HCV infections of blood donors and the general population, we identified transmission risks of TTI that were more prevalent in infected blood donors than in cases from the general population. Their impact was calculated using PAFs for the first time. This indicates challenges for their appropriate identification and for donor education. The largest potential for improvement was found for sexual risk contacts, both heterosexual and MSM (HIV), and piercing/tattoo (HCV), which was also shown for Australia [17]. Although most criteria for (temporary) deferral only target the prevention of window phase donations, an improved recording of transmission risks could reduce the risk of undetected infections among donors. The remarkable impact of effec- 
tive risk-based donor selection and informed donor self-deferral was shown by Yang et al. [18], who estimated a four-fold increase of HIV window period donations in case of donor admission exclusively by NAT.

In addition to donor adherence to deferral policies, the appropriate recognition of sexual risk behavior and subsequent (temporary) deferral of applicant donors seems to be a key measure to avoid TTI, especially HIV infections. However, recording of MSM as well as heterosexual risk behavior remains a challenge and is discussed intensively. In Germany, a uniform DHQ was introduced (for voluntary use), which addressed sexual behavior directly to avoid donors' self-assessment of their infection risk. Pre-evaluation studies showed a higher percentage of correct answers about sexual risk behavior [19] compared to the established DHQs, but a notable portion of donors considered those questions as too personal $[20,21]$. However, it was also shown that questions dealing with sexual risk behaviors were accepted - despite the discomfort - if the questions were demonstrated to potentially increase blood safety [20]. Therefore, in addition to appropriate donor education, the proposed uniform DHQ can help reduce the proportion of donors with sexually transmitted TTI. Sexual risk factors should not only be focused on MSM behavior. Our results showed that heterosexual risk exposures are identified less frequently compared to MSM. In a recent analysis in the UK sex between men and women (SBMW) were frequently reported probable exposures, accounting for $34 \%$ of infections. A new sexual partner was identified as a risk in 4/6 men with SBMW and in 5/6 MSM. Women acquired their infection from new partners (3/9) but also from steady partners who did not disclose their respective risk to the women prior to the blood donation [22]. In a study in the Netherlands incident HBV infections were analyzed. Of the 6 donors, 3 reported a new heterosexual partner, 2 were MSM with recent change of partner, and 1 heterosexual donor had a steady partner with sexual relations outside the steady partnership [23]. Although not all donors are aware of their risk and some donors do not perceive their behavior as risky (e.g. donors in steady partnerships), studies showed that a considerable proportion of infected donors is aware of risk behavior and makes use of the free, sensitive, and confidential testing to check infection status. A study among US donors with sexually transmissible viral infections showed 19\% test-seeking donors [24]; in France 56\% of HIV-infected donors reported test-seeking motivation [25]. In a German case control study, HIV test seeking behavior was significantly more present in HIV-positive donors than in controls [26]. In our opinion, only a comprehensive education of candidate donors about the risks of undetected infectious donations for recipients, other confidential and free test offers and about confidential self-deferral options may help to reduce the risks for TTI which is introduced by test seekers.

A further reduction of TTI might be possible by improved capture of risks apparently related to tattoos and piercings. Although a direct transmission of infections by tattooing/piercing should be excluded due to mandatory hygiene rules, our results correspond to Australian findings [17] where tattoo/piercing prevalence in $\mathrm{HCV}$-infected blood donors was increased four-fold compared to infected persons from the general population. Tattoos were found to be associated with HCV infections even if adjusted for other traditional HCV risk factors [27] but causal evidence is sparse. On the other hand tattoo/piercing is also related to risky sexual behavior and drug use [28] and may in fact be surrogate for undisclosed infection risks. This could also explain our observation of a high percentage of HIV-infected blood donors which reported tattoo/piercing $(55 / 309,18 \%$, included in the category 'others'). Careful assessment of tattoo/piercing of donors is therefore suggested.

Although former imprisonment is rare among blood donors, improved disclosure of this risk factor would have potential to reduce TTI as imprisonment is commonly associated with higher prevalence of infectious diseases [29].

Our study is subject to potential limitations. Infected blood donors have to be notified to the database for infections of blood donors as well as to the national surveillance database for infectious diseases. Due to anonymity of the datasets, infected blood donors could not be extracted from the comparison group (infected persons from the general population), but in view of the much larger comparison group in relation to the infected blood donor population, the introduced bias should be negligible. However, whereas validity of the shown critical risks in blood donors should not be affected by dataset limitations, we could not exclude erroneous rejection of risk factors from multivariable modelling due to underestimation of effects in the analysis.

Furthermore, completeness and reliability of reported risk factors could not be evaluated and might be different between the databases. Some risk factors are prone to nondisclosure due to personal risk perception [5], avoidance of social undesirability, legal consequences, or perceived lack of confidentiality [13] especially in the group of infected blood donors (e.g. sexual risk behavior and drug use). Therefore, estimates for these risk factors were potentially underestimated.

In addition, due to the de-centralized blood establishment system with large proportions of mobile blood drives, not all results from post-donation interviews of blood donors were reported. Therefore, the proportion of infected blood donors with reported risk factors is less complete than that of infected individuals from the general population. However, the sensitivity analysis showed consistency between the analysis of cases with complete recording of transmission risks and the datasets including multiple imputations, especially for HIV infections. Therefore, HIV-infected persons with reported risk factors could be regarded as a representative part of the HIV-infected population. In case of HCV-infected blood donors, relevant underreported risk factors could also be confirmed by our sensitivity analysis, but recording of transmission risks has to be improved substantially to facilitate further analyses.

\section{Conclusion}

The donor selection process should be improved with special attention to the identification of (hetero)sexual risk factors, inva- 
sive procedures like piercing/tattoo, and imprisonment. This could be done by well-designed DHQs, effective donor education, and confidential environment in all steps of the donor selection process.

Moreover, improved activities regarding post-donation recording of TTI infection risks are needed for assessment and probable adaption of currently used DHQ s and specification of deferral criteria. Special attention should be paid to heterosexual risk behavior.

\section{Authorship Contributions}

KP conceived the study, performed the data analysis, and drafted the manuscript, RO initiated the study, supported the interpretation of results, and edited the paper.

\section{Disclosure Statement}

The authors have no competing interests to declare.

\section{References}

1 Slot E, Janssen MP, Marijt-van der Kreek T, Zaaijer HL, van de Laar TJ: Two decades of risk factors and transfusion-transmissible infections in Dutch blood donors. Transfusion 2015;56:203-214.

2 Polizzotto MN, Wood EM, Ingham H, Keller AJ: Reducing the risk of transfusion-transmissible viral infection through blood donor selection: the Australian experience 2000 through 2006. Transfusion 2008;48:55-63.

3 Poethko-Müller C, Zimmermann R, Hamouda O, Faber M, Stark K, Ross RS, Thamm M: Epidemiology of hepatitis $\mathrm{A}, \mathrm{B}$, and $\mathrm{C}$ among adults in Germany: results of the German Health Interview and Examination Survey for Adults (DEGS1) (in German). Bundesgesundheitsbl Gesundheitsforsch Gesundheitsschutz 2013;56:707-715.

4 Robert Koch Institute: Estimates of HIV prevalence and incidence in Germany, status as of end 2014 (in German). Epidemiol Bull 2015:475-486.

5 Grenfell P, Nutland W, McManus S, Datta J, Soldan K, Wellings K: Views and experiences of men who have sex with men on the ban on blood donation: a cross sectional survey with qualitative interviews. BMJ 2011; 343:d5604.

6 Custer B, Sheon N, Siedle-Khan B, Pollack L, Spencer B, Bialkowski W, D’Andrea P, Sullivan M, Glynn S, Williams A: Blood donor deferral for men who have sex with men: the Blood Donation Rules Opinion Study (Blood DROPS). Transfusion 2015;55:2826-2834.

7 Custer B, Kessler D, Vahidnia F, Leparc G, Krysztof DE, Shaz B, Kamel H, Glynn S, Dodd RY, Stramer SL Risk factors for retrovirus and hepatitis virus infections in accepted blood donors. Transfusion 2015;55: 1098-1107.

8 Offergeld R, Kamp C, Heiden M, Norda R, Behr-Gross ME: Sexual risk behaviour and donor deferral in Europe. Vox Sang 2014;107:420-427.

9 Raimondo M, Facco G, Regine V, Pupella S, Grazzini G, Suligoi B: HIV-positive blood donors unaware of their sexual at-risk behaviours before donation in Italy. Vox Sang 2016;110:134-142.

10 Huang Y, Guo N, Yu Q, Lv Y, Ma H, Yun Z, Yao F, Dong X, Zhu X, Wen X, Cao R, Huang M, Bi X, Zhao M, Tiemuer MH, Li J, Zhou Z, He W, Liu J, Wright DJ, Wang J, Ness P, Shan H: Risk factors for hepatitis B and $\mathrm{C}$ infection among blood donors in five Chinese blood centers. Transfusion 2015;55:388-394.
11 Greenland S, Drescher K: Maximum likelihood estimation of the attributable fraction from logistic models. Biometrics 1993;49:865-872.

12 Newson RB: Attributable and unattributable risks and fractions and other scenario comparisons. The Stata Journal 2013;13:672.

13 Lucky TT, Seed CR, Waller D, Lee JF, McDonald A, Wand H, Wroth S, Shuttleworth G, Keller AJ, Pink J, Wilson DP: Understanding noncompliance with selective donor deferral criteria for high-risk behaviors in Australian blood donors. Transfusion 2014;54:17391749.

14 Seed CR, Lucky TT, Waller D, Wand H, Lee JF, Wroth S, McDonald A, Pink J, Wilson DP, Keller AJ: Compliance with the current 12-month deferral for male-tomale sex in Australia. Vox Sang 2014;106:14-22.

15 Wang J, Liu J, Huang Y, Yang T, Yao F, Dong X, Wen G, Bi X, Zhao M, Wen X, Huang M, Lu Y, Ma H, Yu Q, Wright D, Guo N, Ness P, Shan H: An analysis of risk factors for human immunodeficiency virus infection among Chinese blood donors. Transfusion 2013;53: 2431-2440.

16 de Almeida Neto C, McFarland W, Murphy EL, Chen S, Nogueira FA, Mendrone A Jr, Salles NA, Chamone DA, Sabino EC: Risk factors for human immunodeficiency virus infection among blood donors in Sao Paulo, Brazil, and their relevance to current donor deferral criteria. Transfusion 2007;47:608-614.

17 Lucky TT, Seed CR, Keller A, Lee J, McDonald A, Ismay S, Wand H, Wilson DP: Trends in transfusiontransmissible infections among Australian blood donors from 2005 to 2010. Transfusion 2013;53:27512762.

18 Yang H, Anderson SA, Forshee R, Williams A, Epstein JS, Marks PW: Modeling complete removal of risk assessment questions in the USA predicts the risk of HIV exposure in blood recipients could increase despite the use of nucleic acid testing. Vox Sang 2016;110:324-328.

19 Offergeld R, Heiden M, Stoetzer F, Northoff H: Evaluation of a National Blood Donor Questionnaire in Germany. Vox Sang 2010;99:164-165.
20 Sümnig A, Lembcke H, Weber H, Deitenbeck R, Greffin K, Bux J, Greinacher A: Evaluation of a new German blood donor questionnaire. Vox Sang 2014;106:55-60.

21 Weidmann C, Müller-Steinhardt M, Schneider S, Weck E, Klüter H: Donor satisfaction with a new German blood donor questionnaire and intention of the donor to return for further donations. Transfus Med Hemother 2013;40:356-361.

22 Safe Supplies: Uncovering Donor Behavior. Annual Review from the NHS Blood and Transplant/Public Health England Epidemiology Unit, 2014. London, NHS Blood and Transplant/Public Health England Epidemiology Unit, 2015.

23 Lieshout-Krikke RW, Zaaijer HL, van de Laar TJ: Predonation screening of candidate donors and prevention of window period donations. Transfusion 2015; 55:373-378.

24 Vahidnia F, Stramer SL, Kessler D, Goncalez TT, Shaz BH, Leparc G, Krysztof DE, Dodd RY, Glynn SA, Custer B: Motivations for donating and attitudes toward screening policies in US blood donors with viral infection. Transfusion 2016;56:2013-2020.

25 Duquesnoy A, Danic B, Santos A, Martinaud C, Woimant G, Laperche S, Tiberghien P, Jauffret-Roustide $\mathrm{M}$, Pillonel J: Context and social perceptions of blood donation in donors found positive for human immunodeficiency virus in France. Transfusion 2017;57: 2240-2247.

26 Offergeld R, Hamouda O, Burger R: Epidemiological data - an important part of the hemovigilance system. Transfus Med Hemother 2010;37:125-130.

27 Carney K, Dhalla S, Aytaman A, Tenner CT, Francois $\mathrm{F}$ : Association of tattooing and hepatitis $\mathrm{C}$ virus infection: a multicenter case-control study. Hepatology 2013;57:2117-2123.

28 Heywood W, Patrick K, Smith AM, Simpson JM, Pitts MK, Richters J, Shelley JM: Who gets tattoos? Demographic and behavioral correlates of ever being tattooed in a representative sample of men and women. Ann Epidemiol 2012;22:51-56.

29 Fazel S, Baillargeon J: The health of prisoners. Lancet 2011;377:956-965. 